\title{
Selection of transposon mutants of Mycobacterium tuberculosis with increased macrophage infectivity identifies fadD23 to be involved in sulfolipid production and association with macrophages \\ Correspondence \\ Richard W. Stokes \\ rstokes@interchange.ubc.ca \\ Received 6 March 2007 \\ Revised 18 May 2007 \\ Accepted 4 June 2007

\author{
Jennifer Lynett ${ }^{1}$ and Richard W. Stokes ${ }^{2}$
}

\author{
${ }^{1}$ Department of Microbiology and Immunology, University of British Columbia, Vancouver, \\ BC V5Z 4H4, Canada \\ ${ }^{2}$ Departments of Microbiology and Immunology and Paediatrics, University of British Columbia; \\ Division of Infectious and Immunological Diseases, British Columbia's Children's Hospital, \\ Vancouver, BC V5Z 4H4, Canada
}

\section{INTRODUCTION}

The World Health Organization estimates that nearly onethird of the global population is infected with Mycobacterium tuberculosis (Raviglione, 2003). Whilst the currently available antimicrobials are often effective at eradicating the infection, there are issues with patient drug compliance and the emergence of drug-resistant $M$. tuberculosis (Espinal, 2003; Ormerod, 2005). In addition, the rising incidence of HIV infection, particularly in parts of Africa, has further increased the mortality associated with tuberculosis (Nunn et al., 2005). At the onset of infection in the lung, pathogenic mycobacteria gain access to the alveoli, where the bacteria encounter the resident macrophages. While much is known with regard to macrophage receptors that mediate bacterial recognition and subsequent phagocytosis (El-Etr \& Cirillo, 2001; Ernst, 1998), the bacterial surface components that participate in the ligand-receptor interactions remain largely uncharacterized. A thorough understanding of the molecular mechanisms employed by pathogenic mycobacteria when faced with host cells could potentially identify targets for antimicrobial design and lead to the development of novel therapies.
Previously, we reported that disruption of the $M$. tuberculosis surface capsule following sonication significantly increases the level of bacterial association with murine and human macrophages (Stokes et al., 2004). A similar phenotype was also reported following deletion of the pks3-pks4 locus of $M$. tuberculosis, which reduced the retention of the surface capsule due to a deficiency in mycosanoic and mycolipenic acid synthesis that resulted in the absence of diacyltrehaloses and polyacyltrehaloses in the cell envelope (Dubey et al., 2002; Rousseau et al., 2003). Increased bacterial recognition by macrophages has also been demonstrated in non-tuberculous mycobacteria lacking surface glycopeptidolipids (Etienne et al., 2002). Together these findings suggest that modifications to the mycobacterial cell wall can potentially lead to the exposure of bacterial ligands that are readily recognized by macrophages. Interestingly, a recent study of $M$. tuberculosis clinical isolates reported that strains associated with extrarespiratory sites of infection correlated with an increased level of macrophage infectivity, suggesting that the initial interactions with the host macrophage directly affect bacterial pathogenesis (Garcia de Viedma et al., 2005).

The focus of the present study was to identify and characterize mycobacterial factors that participate in the initial binding interaction with host macrophages. A transposon library of $M$. tuberculosis was screened for mutants that showed an increased level of macrophage 
association as compared to the parental wild-type. Our expectation was that this screening strategy would identify mycobacterial genes that contribute, in some manner, to the biogenesis of the cell wall. A total of five mutants were selected and the sites of transposon disruptions were localized to loci encoding enzymes involved in lipid biosynthesis as well as hypothetical proteins. One mutant containing a transposon disruption within the fadD23 gene was repeatedly isolated from the library. Lipid analysis of the $f a d D 23$ mutant indicated a deficiency in the production of cell wall sulfolipids. Genetic complementation of the transposon mutant with the wild-type gene led to restoration of the wild-type phenotype for both macrophage binding and sulfolipid production.

\section{METHODS}

Bacterial strains. M. tuberculosis Erdman (ATCC 35801) was grown in Middlebrook $7 \mathrm{H} 9$ broth (Difco) supplemented with $0.5 \%$ glycerol, $10 \%$ oleic acid/albumin/glucose (dextrose) complex (OADC) and $0.05 \%$ Tween or on Middlebrook 7H10 agar (Difco) supplemented with $0.5 \%$ glycerol and $10 \%$ OADC. OADC was prepared as described previously (Dullaghan et al., 2002). For the preparation of lipid extracts, mycobacteria were cultured in glycerol/alanine/salts (GAS) medium (Takayama et al., 1975). Escherichia coli strains were grown in Luria-Bertani (LB) broth or on LB-agar plates. When required, hygromycin and kanamycin were used at concentrations of $50 \mu \mathrm{g} \mathrm{ml}^{-1}$ and $25 \mu \mathrm{g} \mathrm{ml}^{-1}$ respectively.

Transposon mutagenesis. A transposon library was generated using the pHAE87 vector and Tn5370 as described previously (Bardarov et al., 1997). Briefly, a $100 \mathrm{ml}$ culture of M. tuberculosis was grown to $\mathrm{OD}_{600}$ 0.8-1.0. The cells were washed twice and resuspended in a final volume of $10 \mathrm{ml}$ phage adsorption buffer (10 mM Tris/HCl, pH 7.6, $100 \mathrm{mM} \mathrm{NaCl}, 10 \mathrm{mM} \mathrm{MgSO}_{4}, 2 \mathrm{mM}$ $\mathrm{CaCl}_{2}$ ). The bacteria were infected with the phage lysate at an m.o.i. of 10:1 (phage particles:bacteria) for $4 \mathrm{~h}$ at $39^{\circ} \mathrm{C}$. The infected cell suspension was centrifuged at $16000 \mathrm{~g}$ for $5 \mathrm{~min}$ to recover the bacteria before plating onto selective media. After 4-6 weeks of growth, hygromycin-resistant colonies were scraped from the agar plates and cultured in $7 \mathrm{H} 9$ in separate pools each containing roughly 500 mutants. After 7-10 days of growth, the cultures were aliquoted and stored at $-80{ }^{\circ} \mathrm{C}$. A total of 20 library pools were used in the screening experiments.

Southern blot analysis on a total of 38 randomly selected mutants confirmed that significant diversity in mutant genotypes was present in the transposon library (data not shown). Briefly, genomic DNA from the 38 mutants was digested with $P v u I I$, which does not cut within the transposon, and transferred onto nylon membrane as described elsewhere (Sambrook et al., 1989). The blots were hybridized with a DIG-labelled DNA fragment derived from the hygromycin-resistance cassette according to the directions of the manufacturer (Roche Applied Science). The insertion patterns and limitations of Tn5370 transposon have been previously characterized (McAdam et al., 2002). Thus, while the library may not be representative of the whole genome, it is likely that insertions are present in a significant number of the genes.

Infection of THP-1 cells. The human macrophage-like cell line THP-1 (ATCC TIB202) was cultured in RPMI 1640 (Gibco) supplemented with $10 \%$ heat-inactivated fetal calf serum (Gibco), $10 \mathrm{mM}$ L-glutamine (Gibco) and $10 \mathrm{mM}$ sodium pyruvate (Gibco) and maintained in an atmosphere of $5 \% \mathrm{CO}_{2}$ at $37{ }^{\circ} \mathrm{C}$ as described previously (Stokes \& Doxsee, 1999). For infection assays, THP-1 cells were seeded onto acid-washed glass coverslips $\left(2 \times 10^{5}\right.$ cells per coverslip) or into T-75 $\mathrm{cm}^{2}$ flasks $\left(5 \times 10^{6}\right.$ cells $)$ and differentiated in the presence of $100 \mathrm{nM}$ phorbol 12-myristate 13-acetate (PMA; Sigma-Aldrich) over 3 days. Prior to infection, the monolayers were washed with binding medium to remove any residual PMA.

Single-cell suspensions of mycobacteria for use during infection assays were prepared using two separate techniques, essentially as described previously (Stokes et al., 2004). Briefly, the bacteria were pelleted by centrifugation (16 $000 \mathrm{~g}$ for $5 \mathrm{~min}$ ) and resuspended in $500 \mu$ binding medium $\left(138 \mathrm{mM} \mathrm{NaCl}, 8.1 \mathrm{mM} \mathrm{Na}_{2} \mathrm{HPO}_{4}, 1.5 \mathrm{mM}\right.$ $\mathrm{KH}_{2} \mathrm{PO}_{4}, 2.7 \mathrm{mM} \mathrm{KCl}, 0.6 \mathrm{mM} \mathrm{CaCl}, 1 \mathrm{mM} \mathrm{MgCl}_{2}$ and $5.5 \mathrm{mM} \mathrm{D}-$ glucose; Smith \& Iden, 1981). The bacterial clumps were then dispersed by 10 repeated passages through a 25 -gauge needle attached to a $1 \mathrm{ml}$ syringe or by three $30 \mathrm{~s}$ pulses of bath sonication using a VC50T $50 \mathrm{~W}$ microcup horn (Sonics \& Materials) as indicated for each experiment (Stokes et al., 2004). The volume of the cell suspension was then adjusted using binding medium to achieve the desired bacterial concentration.

The differentiated THP-1 cells were infected with mycobacteria for two purposes: (i) to screen the transposon library and (ii) to test the macrophage binding affinities for the mutant clones selected for further study. For procedure (i), THP-1 cells cultured in T-75 $\mathrm{cm}^{2}$ flasks and on coverslips were infected with the library pools (syringed suspensions) at an m.o.i. of 100:1 (bacteria:macrophages) for $4 \mathrm{~h}$. Non-adherent mycobacteria were removed by vigorously washing the monolayer three times with binding medium $(10 \mathrm{ml}$ for flasks, $500 \mu \mathrm{l}$ for coverslips), a method that we have previously shown to be effective for the removal of non-adherent bacteria (Stokes et al., 1986). The infected coverslips were fixed (10 $\mathrm{min}$ in $10 \%$ formaldehyde in ethanol) and mounted onto microscope slides followed by staining with Kinyoun's Carbol Fuchsin and malachite green. The level of bacterial binding for each mutant pool was determined by microscopic count. The infected monolayers in the T$75 \mathrm{~cm}^{2}$ flasks were used to enrich for mutants with enhanced hostcell-binding capabilities; the THP-1 cells were dislodged using a cell scraper, resuspended in PBS containing $0.1 \%$ Tween-80 (PBS-Tween) and briefly sonicated to lyse the macrophages. The macrophageassociated bacteria were recovered and cultured for 7-10 days in $7 \mathrm{H} 9$ before being used as the inoculum in a subsequent round of selection. After six to eight rounds of enrichment, the library pools that displayed an increased level of macrophage association (as determined by microscopic count) were plated onto selective agar plates and individual mutant clones were assessed for macrophage infectivity.

For procedure (ii), syringed and sonicated suspensions of mycobacteria were prepared as described above and used to infect THP-1 monolayers on coverslips at an m.o.i. of $100: 1$ (bacteria:macrophages) for $3 \mathrm{~h}$. The monolayers were fixed and stained as described above. The percentage of infected macrophages was determined by counting 100 macrophages for each coverslip.

Identification of the transposon insertion site. Genomic DNA from the selected transposon mutants was extracted (Belisle \& Sonnenberg, 1998), restriction digested with PvuII and analysed by Southern hybridization using a labelled fragment from the hygromycin-resistance cassette. The presence of a single hybridizing DNA fragment on the Southern blot verified that a single copy of the transposon had incorporated into the chromosome. The precise location of the transposon insertion was determined by sequencing the amplicons generated by ligation-mediated inverse PCR (Cox et al., 1999). Briefly, $2.5 \mu \mathrm{g}$ DNA was digested with RsaI. The restriction digest was extracted once with phenol/chloroform/isoamyl alcohol $(25: 24: 1)$ and precipitated with ethanol. An aliquot of the digested DNA ( $200 \mathrm{ng})$ was ligated overnight at $16{ }^{\circ} \mathrm{C}$, followed by ethanol 
precipitation. The DNA sequences adjacent to the transposon were amplified using primers corresponding to the left (O84L-F 5'-GTCATCCGGCTCATCACCAG-3' and O84L-R 5'-AACTGGCGCAGTTCCTCTGG-3') and right (O84R-F 5'-ATACACGCGCACCGGTTCTAGC-3 and O84R-R 5'-CACGGCGAACCGCTGGTG-3') ends of the transposon. Sequence comparisons were done using BLAST 2.0 at www.ncbi.nlm.nih.gov/. The transposon insertion within the fadD23 gene was also confirmed by PCR using primers that flank the site of transposon integration; the FADD-L 5'-TCTGACAACATCCGCGATAA-3' primer corresponds to nucleotides 348368 and the FADD-R 5'-GTCACTTCGAAGGCGAAGTT-3' primer matches nucleotides $807-827$ of the fadD23 gene.

Complementation of the fadD23 transposon mutant. The complementation construct was generated by amplifying the fadD23 gene along with $455 \mathrm{bp}$ of upstream sequence from M. tuberculosis chromosomal DNA using Pfu DNA polymerase (Stratagene) with the following primers (FADJLL 5'-CGTAGAACTCGTCGCAATCC-3' and FADJLR 5'-GTGGATCCGACCGTAAGACC-3'). The amplicon was blunt-end ligated into the mycobacterial integration vector pMV306, which had been digested with EcoRV (Stover et al., 1991). The resulting plasmid construct was sequenced and transformed into the mutant by electroporation as described elsewhere (Parish \& Stoker, 1998). Transformed bacteria were recovered using kanamycin selection and the presence of the wild-type fadD23 gene in the complemented mutant was confirmed by PCR using the FADD-L and FADD-R primers.

Lipid analysis. Apolar and polar lipids were extracted from bacterial cultures that were pelleted by centrifugation, autoclaved $\left(121{ }^{\circ} \mathrm{C}\right.$, $60 \mathrm{~min}$ ) and subsequently lyophilized as described previously (Besra, 1998). Lipids were separated by two-dimensional thin-layer chromatography (2D-TLC) on Silica Gel 60 plates (EM Science) using five different solvent systems (A-E) for mycobacterial lipid analysis that have been described elsewhere (Besra, 1998; Dobson et al., 1985). Sulfolipids were localized using solvent system D, which uses chloroform/methanol/water $(100: 14: 0.8)$ for the first-dimension solvent system and chloroform/acetone/methanol/water $(50: 60: 2.5: 3)$ for the second dimension. Lipids were visualized by spraying TLC plates with either $5 \%$ ethanolic phosphomolybdic acid or $2.5 \% \quad \alpha$-naphthol $/ 10 \%$ sulfuric acid followed by charring. Sulfolipid 1 (SL-1) was also detected by staining TLC plates with cresyl violet as described elsewhere (Soto et al., 2000). Briefly, the lipids were resolved on TLC plates using solvent system D and the plates were immersed in a solution of $0.02 \%$ cresyl violet in $1 \%$ aqueous acetic acid for $10 \mathrm{~min}$ followed by briefly destaining with $1 \%$ aqueous acetic acid (data not shown).

Statistical analysis. The results shown are the mean \pm SEM. Statistically significant differences between groups were determined with the two-tailed, unpaired Student's $t$ test or analysis of variance. $P$ values $<0.05$ were considered significant.

\section{RESULTS}

\section{Transposon mutagenesis and mutant selection}

A transposon library using $M$. tuberculosis Erdman was generated using the Tn5370 transposon in conjunction with a temperature-sensitive mycobacteriophage delivery vector (Bardarov et al., 1997). A biological screening assay consisting of iterative rounds of macrophage infection was used to select for transposon mutants with an enhanced ability to bind macrophages. As outlined in Fig. 1, 20 pools from a transposon library (each containing $~ 500$ mutants) of $M$. tuberculosis were used to infect THP-1 cell monolayers. Transposon mutants that preferentially associated with host cells were recovered from the monolayer and used as the initial inoculum in a subsequent macrophage infection assay. We hypothesized that the desired phenotype would become enriched within the output mutant population after successive rounds of selection. Between the sixth and eighth round of successive macrophage infection assays, three of the library pools emerged that consistently bound the cell monolayers more readily than the syringed $M$. tuberculosis control (Fig. 2). The three pools were plated onto $7 \mathrm{H} 10$ agar and 40 mutants from each pool were isolated at random. Southern blotting was used to identify siblings within each pool (data not shown) and the level of macrophage association was determined for each of the 40 mutants from the three pools (a total of 120 mutants). The site of transposon insertion was identified using inverse PCR and DNA sequencing of mutants within the pools with the highest levels of macrophage association (Table 1). None of the mutants appeared to have any alterations in colony morphology or growth rate in broth when compared to the wild-type (data not shown).

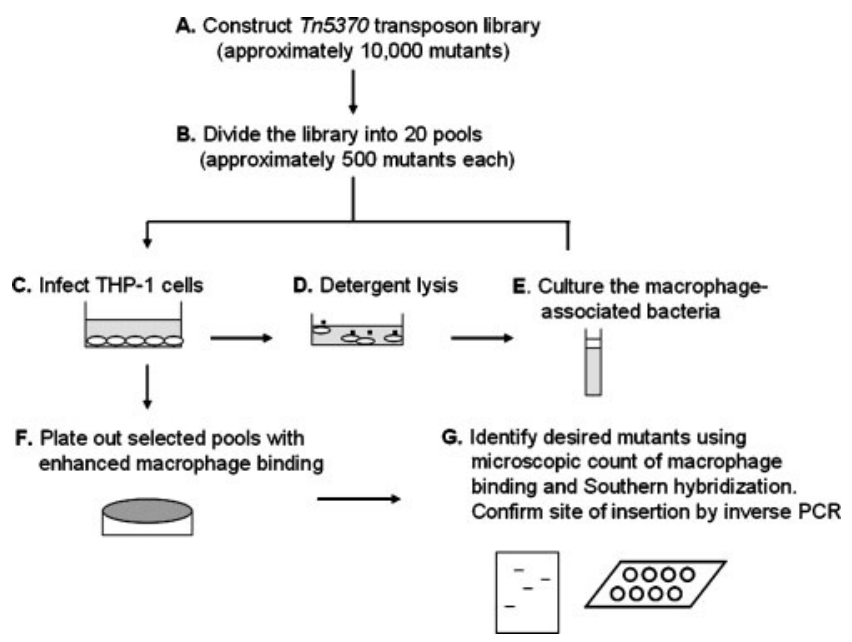

Fig. 1. Library screening to identify mutants with an increased macrophage binding affinity. (A) A transposon library was generated using the Tn5370 transposon. (B) The library was subdivided into 20 pools each containing 500 mutants. (C) The mutant pools were serially passaged using binding to THP-1 cells as the selection criterion. (D) Cell-associated bacteria were recovered from the monolayer. (E) These bacteria were cultured in broth and used as the infecting inoculum in the next round of selection. (F) Three mutant pools that showed increased levels of macrophage binding were plated onto $7 \mathrm{H} 10$ agar. (G) Mutants from each of the selected pools were tested for their ability to bind macrophages and Southern hybridization was used to identify siblings. The site of transposon insertion was determined by inverse PCR. 

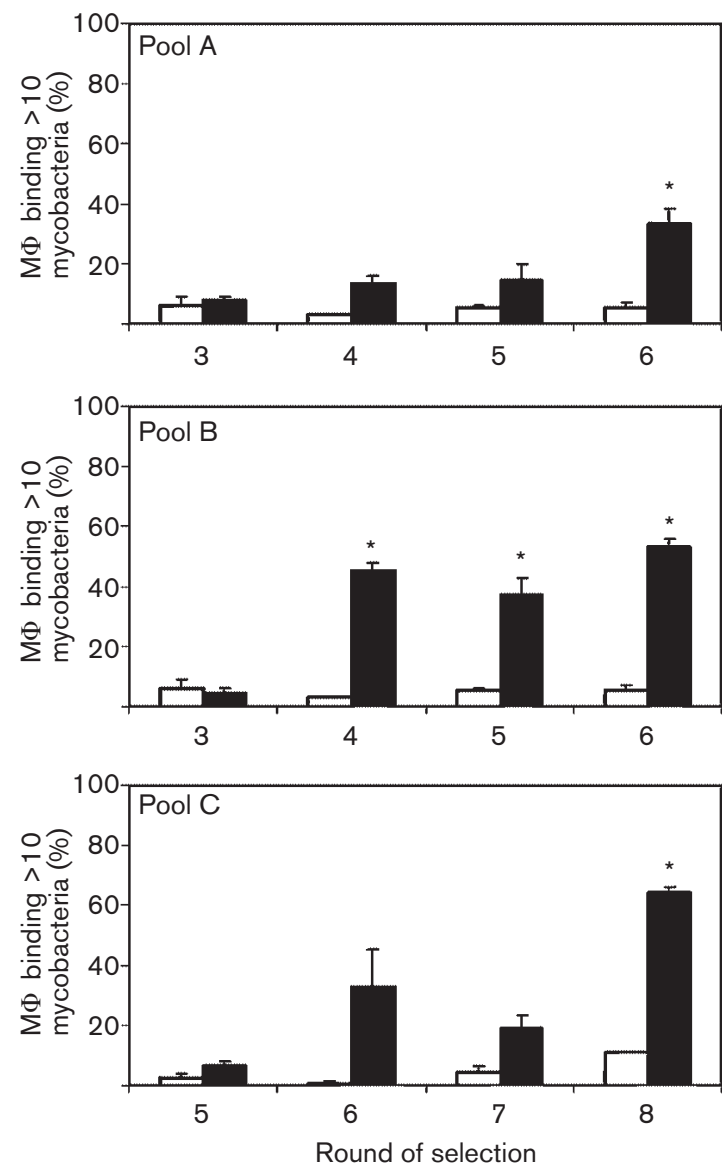

Fig. 2. Pools of mutants that exhibit increasing levels of macrophage association. During library screening, three mutant pools $(A-C)$ were identified that showed increasing levels of macrophage association with each round of selection. The level of macrophage binding was determined by microscopic count of fixed monolayers. The graphs represent the percentage of macrophages binding $>10$ syringe-dispersed (see Methods) M. tuberculosis (white bars) or $>10$ syringe-dispersed bacteria from the library pool (black bars). The data shown are the means \pm SEM of three infected coverslips. * denotes $P<0.05$ when compared to syringedispersed wild-type M. tuberculosis.

As shown in Fig. 3, all of the mutants identified from the selection procedure exhibited a binding phenotype that more closely mimicked that of the sonicated preparation of wild-type M. tuberculosis. These increases in macrophage infectivity suggested that the transposon insertions led to an altered bacterial surface and increased recognition by the host macrophages. To confirm that the increases in bacterial binding were not merely a side effect of the mutagenesis procedure, we included a transposon mutant (mutant 1) from library pool A as a negative control. As shown in Fig. 3, the mutant 1 strain did not exhibit any increase in macrophage association and was indistinguishable from a syringed preparation of wild-type $M$. tuberculosis, suggesting that the phenotypes of the mutants identified from the screening process could not be simply attributed to the transposon construct. It is also important to note that the transposon mutants that were identified from the screening procedure did not exhibit any increased bacterial clumping when cultured in vitro and that the syringe-dispersal treatment used prior to macrophage infection was sufficient for the disruption of small clumps of mycobacteria, as previously described (Stokes et al., 2004)

\section{Lipid analysis of the fadD23 : : Tn5370 mutant}

Among the five transposon mutants that were identified from the library screen, the fadD23 transposon mutant was repeatedly isolated from two of the three library pools that demonstrated an enhanced macrophage-binding phenotype: the transposon disruption in $f a d D 23$ was detected in five of the 40 mutants from pool A tested by Southern analysis and two of 40 mutants from pool B. The transposon insertion mapped to nucleotide 630 of the fadD23 gene, shown in Fig. 4(a). The fadD23 mutant displayed an increased binding affinity to THP-1 cells as compared to wild-type $(P<0.05$, ANOVA $)$. Complementation of the mutation with the fadD23 gene in trans resulted in binding levels that were intermediate between the wild-type and the mutant, and not significantly different from either $(P>0.05$, ANOVA), indicating that partial restoration of the binding phenotype was achieved following complementation (Fig. 4b). The close proximity of the $f a d D 23$ gene to other loci that have been linked to sulfolipid biosynthesis prompted us to investigate whether the biosynthesis of cell wall glycolipids was affected in the fadD23 transposon mutant. The apolar and polar lipid fractions were extracted from whole-cell pellets of the parental wild-type, the mutant and the complemented strain for analysis by TLC. As shown in Fig. 5, comparison of the apolar lipid fractions revealed a lack of sulfolipid production in the absence of $f a d D 23$. Confirmation that the lipid fraction was indeed sulfolipid was obtained by staining the TLC plates with cresyl violet (Soto et al., 2000) (data not shown). Importantly, sulfolipid production could be restored following complementation of the mutant with the wild-type fadD23 gene.

\section{DISCUSSION}

To date much of the research surrounding the interaction between $M$. tuberculosis and host macrophages has centred on the host-cell receptors that mediate bacterial internalization. Furthermore, while great strides have been made in elucidating the chemical composition of the mycobacterial cell envelope, much less is known about the biological function of the different cell wall moieties during infection. Previous findings from our group have indicated that certain mechanical treatments that are used to generate single-cell suspensions of mycobacteria affect both the 
Table 1. Mutants isolated from the transposon library that exhibit an increased binding affinity to THP-1 cells

\begin{tabular}{|llcl|}
\hline $\begin{array}{l}\text { M. tuberculosis } \\
\text { H37Rv gene ID }\end{array}$ & Gene name & $\begin{array}{c}\text { Site of insertion/location } \\
\text { on genome (nucleotides)* }\end{array}$ & \multicolumn{1}{c|}{ Predicted function } \\
\hline Rv3826 & $f a d D 23$ & $+630 / 4300442$ & Fatty-acyl AMP ligase \\
Rv1505c & $R v 1505 c$ & $+335 / 1695611$ & Conserved hypothetical protein \\
Rv0405 & $p k s 6$ & $+1237 / 486968$ & Polyketide synthase \\
Rv3335c & $R v 3335 c$ & $+663 / 3721937$ & Probable conserved integral membrane protein \\
Rv1115 & $R v 1115$ & $-28 / 1240159$ & Possible exported protein \\
& & & \\
\hline
\end{tabular}

${ }^{*}$ The site of insertion is relative to the coding sequence of the gene as determined using Tuberculist (http:// genolist.pasteur.fr/TubercuList/). The location on the genome is relative to the initiation codon of the $d n a A$ gene as described previously (Cole et al., 1998).

bacillus surface and the level of interaction with host macrophages (Stokes et al., 2004). We hypothesized that this observation could be used as a library screening tool to identify mutants that had incurred cell wall alterations as a result of mutagenesis. In this investigation, we screened a transposon library of $M$. tuberculosis using successive rounds of infection assays with THP-1 cell monolayers. Five mutants were identified from the screen that exhibited an increased binding affinity for host cells as compared to the parental wild-type, and at least three of these disrupted

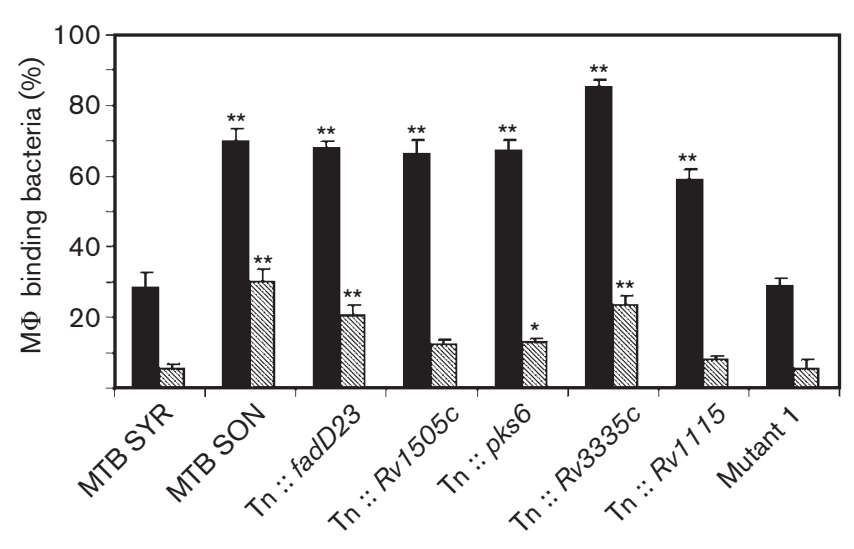

Fig. 3. Macrophage binding of mutants identified by library selection. The individual mutant clones (fadD23::Tn5370, Rv1505c::Tn5370, pks6::Tn5370, Rv3335c::Tn5370 and Rv1115::Tn5370) were cultured to exponential phase and syringe-dispersed (see Methods) prior to infecting THP-1 macrophages. Wild-type M. tuberculosis, either syringe-dispersed (MTB SYR) or sonicated (MTB SON), were included as controls. A transposon mutant from the library (mutant 1 ) was also included as a control for non-specific effects of the transposon. Black bars represent the percentage of macrophages associated with at least one bacterium; hatched bars represent the percentage of macrophages associated with $>10$ bacteria. The mean \pm SEM is shown for two independent experiments each with triplicate coverslips. ${ }^{\star} P<0.05,{ }^{* \star} P<0.001$ when compared to syringedispersed wild-type M. tuberculosis. genes ( $R v 3335 c$, pks6 and $R v 1115$ ) have been previously linked to mycobacterial pathogenesis.

The $p k s 6$ gene encodes a predicted polyketide synthase that was initially identified by signature-tagged mutagenesis and mutants in this gene were found to be impaired for growth in the lungs of Balb/c mice (Camacho et al., 1999). Separate studies have also suggested that Pks6 is involved in countering host defences that involve interferon- $\gamma$ (Hisert et al., 2004). Although the precise biological function of Pks6 is unknown, a recent report suggests its involvement in the synthesis of polar lipids within the M. tuberculosis cell envelope (Waddell et al., 2005). The Rv1115 and $R v 3335 c$ genes encode hypothetical proteins for which there is little functional information, although these loci have been previously identified from separate transposon libraries; a transposon mutant in $R v 3335 c$ has been previously shown to be defective for growth within the spleens of C57/BL6 mice using the transposon-site hybridization (TRASH) system (Sassetti et al., 2003) and disruption of the Rv1115 gene in Mycobacterium bovis BCG leads to increased accumulation of the mutant within acidified phagosomes following macrophage infection (Stewart et al., 2005). The Rv1505c gene encodes a predicted protein with a conserved domain that is associated with enzymes belonging to the hexapeptide repeat superfamily of acetyltransferases (Raetz \& Roderick, 1995; Vuorio et al., 1994; Wenzel et al., 2005). It is important to note that confirmation of the phenotypes for the Rv3335c, pks6, Rv1115 and Rv1505c mutants will require genetic complementation to rule out the possibility of polar effects. In particular, the ORFs downstream from the $R v 1505 c$ and $R v 1115$ genes may be affected as a result of the transposon insertions. The $R v 1505 c$ gene appears to reside within an operon that encompasses $R v 1505 c-$ $R v 1503 c$. The $R v 1503 c$ and $R v 1504 c$ genes, which are immediately downstream of $R v 1505 c$, belong to the DegT/ DnrJ/EryC1/StrS aminotransferase family in the protein families (Pfam) database (Bateman et al., 2004). Interestingly, the Rv1503-Rv1504 proteins share a high degree of similarity with the WecE protein (59\% identity, $71 \%$ similarity) of E. coli and Salmonella enterica. Within 
(a)

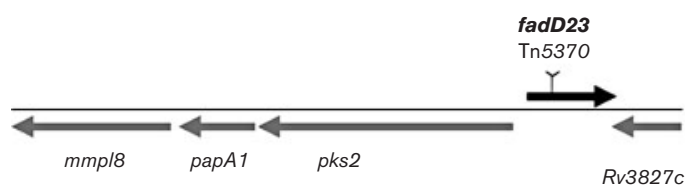

(b)

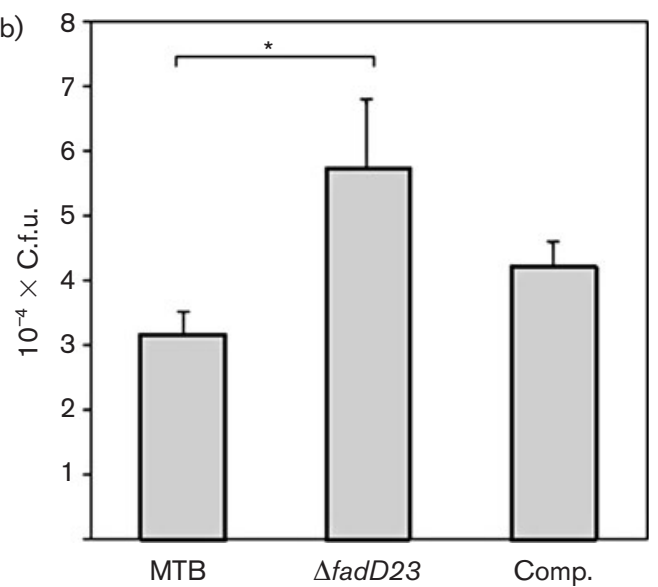

Fig. 4. Disruption of the fadD23 gene results in increased association between $M$. tuberculosis and macrophages. (a) Diagram of the genomic region that encompasses the fadD23 gene, showing the site of transposon mutagenesis. (b) Association of wild-type M. tuberculosis (MTB), fadD23::Tn5370 and the complemented strain (Comp.) with THP-1 monolayers. The mean \pm SEM is shown for three independent experiments, each with triplicate coverslips. ${ }^{*} P<0.05$ when comparing the fadD23 mutant to wild-type M. tuberculosis.

these enteric pathogens, WecE is a sugar aminotransferase that functions in the synthesis of the carbohydrate component of the enterobacterial common antigen, a glycolipid located on the outer leaflet of the Gram-negative outer membrane. In the case of the $R v 1115$ transposon mutant, the downstream locus (Rv1116) is located just over $100 \mathrm{bp}$ downstream and encodes a hypothetical protein of unknown function. Polar effects from the transposon are less likely to be an issue with the $p k s 6$ and $R v 3335 c$ mutants since the downstream loci are not cotranscribed in the same orientation.

The fadD23 transposon mutant was chosen for further study as this mutation was repeatedly identified within the output population of mutants that remained after the selection assays. The fadD23 gene is located upstream of two loci that have been confirmed to be involved in sulfolipid biosynthesis. The Pks2 polyketide synthase catalyses the formation of the sulfolipid acyl chains (Sirakova et al., 2001) while the MmpL8 protein mediates the transport of mature sulfolipid or its precursors from the cytosol into the cell envelope (Converse et al., 2003; Domenech et al., 2004). The fadD23 gene probably encodes a fatty-acyl CoA ligase that would function in concert with the Pks2 enzyme to generate the glycolipid acyl chains (Trivedi et al., 2004). The fadD23 gene is flanked on the other side by the $R v 3827 c$ gene, which encodes a putative transposase. It is unlikely that polar effects play a role in the phenotype of the fadD23 mutant given that the transcription of the $R v 3827 c$ gene is in the opposite orientation relative to $f a d D 23$ and that mutant complementation was sufficient to restore the wild-type phenotype. Thus from the data presented in this report, we propose that the FadD23 protein should now also be included in the list of SL-1 biosynthetic enzymes. Comparative TLC analysis of the apolar lipid fractions from the parental wild-type and the fadD23 transposon mutant confirmed the absence of mature SL-1 on the cell surface of the mutant strain. In addition, SL-1 production could be restored by complementation of the mutant with the fadD23 gene. We found that disruption of the $f a d D 23$ gene resulted in an increased level of bacterial association with macrophages which was restored to wild-type levels following complementation.

The correlation of SL-1 loss with increased binding of $M$. tuberculosis to macrophages could reflect alterations in the

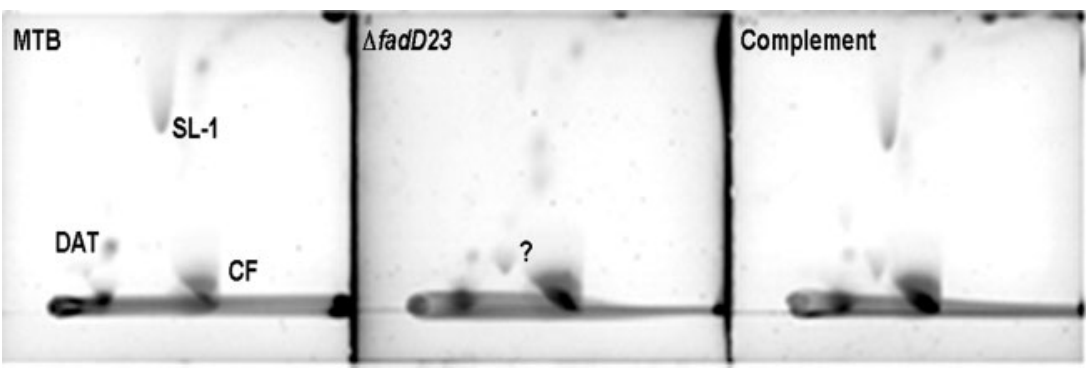

Fig. 5. Disruption of fadD23 in MTB leads to a loss of SL-1 production. Apolar lipids from $M$. tuberculosis (MTB), fadD23: :Tn5370 and the complemented strain (Complement) were separated by 2D-TLC to resolve SL-1. Plates were stained with $\alpha$-naphthol and charred. DAT, diacyltrehalose; CF, cord factor; SL-1, sulfolipid-1; ?, unknown lipid. 
organization of the cell envelope leading to the exposure of previously masked bacterial ligands. Alternatively, the block in sulfolipid production seen in the mutant may lead to an upregulation in the synthesis of other cell wall lipids. Recently, Jain et al. (2007) reported that PDIM mutants of $M$. tuberculosis synthesized an increased abundance of sulfolipid due to the increased availability of methylmalonyl-CoA precursors. While the authors did not identify a reciprocal increase in PDIM synthesis in SL-1 deficient mutants (Jain et al., 2007), there remains the possibility that the transposon mutation in fadD23 may cause a disturbance in the total distribution of cell wall lipids. Finally, the absence of SL-1 may also affect the overall surface charge of the bacillus, thereby increasing the level of cell-cell interactions. In support of this possibility, we have previously shown a correlation between a negatively charged mycobacterial surface and enhanced macrophage association (Stokes et al., 2004).

There are other examples in the literature where loss of cell surface lipids has resulted in improved association of mycobacteria and host cells. Mycobacterium smegmatis mutants lacking cell wall glycopeptidolipids (GPL) are more rapidly internalized by macrophages than are wild-type bacteria (Etienne et al., 2002), perhaps due to the inhibitory effects of GPL on M. smegmatis binding (Villeneuve et al., 2003). In addition, inactivation of the $p k s 3-p k s 4$ locus in $M$. tuberculosis coincides with an increase in macrophage binding due to loss of diacyltrehaloses and polyacyltrehaloses (Rousseau et al., 2003). Collectively, these and our earlier findings (Stokes et al., 2004) suggest that the interaction of mycobacteria with macrophages is limited by the presence of an intact outer cell envelope, disruption of which leads to enhanced binding.

In summary, transposon mutants were identified that were more apt to associate with phagocytic cells, implying that these strains had sustained some cell surface alterations responsible for their increased uptake. Lipid analysis of the fadD23 transposon mutant confirmed the involvement of fadD23 in the synthesis of cell wall sulfolipid. However, it is important to note that the collection of mutants identified in this study does not represent an exhaustive list of all the mycobacterial genes involved in binding to host macrophages. In particular, the use of large pools of mutants as part of the screening process does not favour the selection of mutant clones that have reduced rates of in vitro replication. There is also the possibility that neighbouring bacteria may complement the phenotypes of other mutants within the same pool (Sassetti \& Rubin, 2003). Further investigation will focus on the cell wall compositions of the remaining mutants and will determine whether the alterations in the cell envelope correlate with changes in bacterial virulence.

\section{ACKNOWLEDGEMENTS}

This work was supported by a Canadian Institutes of Health Research grant (MOP-43924 to R. W.S.) and R. W.S. is the recipient of a Child and Family Research Institute Investigatorship Award. J. L. was supported by a University Graduate Fellowship from the University of British Columbia and a BC Research Institute for Children's and Women's Health Graduate Studentship. We would like to thank W. R. Jacobs Jr, Albert Einstein College of Medicine, New York, for providing us with the transposon mutagenesis system for use in mycobacteria and K. G. Papavinasasundaram, Dept of Medicine, University of British Columbia, for providing the pMV306 integration vector.

\section{REFERENCES}

Bardarov, S., Kriakov, J., Carriere, C., Yu, S., Vaamonde, C., McAdam, R. A., Bloom, B. R., Hatfull, G. F. \& Jacobs, W. R., Jr (1997). Conditionally replicating mycobacteriophages: a system for transposon delivery to Mycobacterium tuberculosis. Proc Natl Acad Sci U S A 94, 10961-10966.

Bateman, A., Coin, L., Durbin, R., Finn, R. D., Hollich, V., GriffithsJones, S., Khanna, A., Marshall, M., Moxon, S. \& other authors (2004). The Pfam protein families database. Nucleic Acids Res 32, D138-D141.

Belisle, J. T. \& Sonnenberg, M. G. (1998). Isolation of genomic DNA from mycobacteria. In Methods in Molecular Biology: Mycobacteria Protocols, pp. 31-44. Edited by T. Parish \& N. G. Stoker. Totowa, NJ: Humana Press.

Besra, G. S. (1998). Preparation of cell wall fractions from mycobacteria. In Methods in Molecular Biology: Mycobacteria Protocols, pp. 91-107. Edited by T. Parish \& N. G. Stoker. Totowa, NJ: Humana Press.

Camacho, L. R., Ensergueix, D., Perez, E., Gicquel, B. \& Guilhot, C. (1999). Identification of a virulence gene cluster of Mycobacterium tuberculosis by signature-tagged transposon mutagenesis. Mol Microbiol 34, 257-267.

Cole, S. T., Brosch, R., Parkhill, J., Garnier, T., Churcher, C., Harris, D., Gordon, S. V., Eiglmeier, K., Gas, S. \& other authors (1998). Deciphering the biology of Mycobacterium tuberculosis from the complete genome sequence. Nature 393, 537-544.

Converse, S. E., Mougous, J. D., Leavell, M. D., Leary, J. A., Bertozzi, C. R. \& Cox, J. S. (2003). MmpL8 is required for sulfolipid-1 biosynthesis and Mycobacterium tuberculosis virulence. Proc Natl Acad Sci U S A 100, 6121-6126.

Cox, J. S., Chen, B., McNeil, M. \& Jacobs, W. R., Jr (1999). Complex lipid determines tissue-specific replication of Mycobacterium tuberculosis in mice. Nature 402, 79-83.

Dobson, G., Minnikin, D. E., Minnkin, S. M., Pharlett, J. H., Goodfellow, M., Ridell, M. \& Magnusson, M. (1985). Systematic analysis of complex mycobacterial lipids. In Chemical Methods in Bacterial Systematics, pp. 237-265. Edited by M. Goodfellow \& D. E. Minnikin. London: Academic Press.

Domenech, P., Reed, M. B., Dowd, C. S., Manca, C., Kaplan, G. \& Barry, C. E., III (2004). The role of MmpL8 in sulfatide biogenesis and virulence of Mycobacterium tuberculosis. J Biol Chem 279, 21257-21265.

Dubey, V. S., Sirakova, T. D. \& Kolattukudy, P. E. (2002). Disruption of msl3 abolishes the synthesis of mycolipanoic and mycolipenic acids required for polyacyltrehalose synthesis in Mycobacterium tuberculosis H37Rv and causes cell aggregation. Mol Microbiol 45, 1451-1459.

Dullaghan, E. M., Malloff, C. A., Li, A. H., Lam, W. L. \& Stokes, R. W. (2002). Two-dimensional bacterial genome display: a method for the genomic analysis of mycobacteria. Microbiology 148, 3111-3117.

El-Etr, S. H. \& Cirillo, J. D. (2001). Entry mechanisms of mycobacteria. Front Biosci 6, D737-D747. 
Ernst, J. D. (1998). Macrophage receptors for Mycobacterium tuberculosis. Infect Immun 66, 1277-1281.

Espinal, M. A. (2003). The global situation of MDR-TB. Tuberculosis (Edinb) 83, 44-51.

Etienne, G., Villeneuve, C., Billman-Jacobe, H., Astarie-Dequeker, C., Dupont, M. A. \& Daffe, M. (2002). The impact of the absence of glycopeptidolipids on the ultrastructure, cell surface and cell wall properties, and phagocytosis of Mycobacterium smegmatis. Microbiology 148, 3089-3100.

Garcia de Viedma, D., Lorenzo, G., Cardona, P. J., Alonso Rodriguez, N., Gordillo, S., Ruiz Serrano, M. J. \& Bouza, E. (2005). Association between the infectivity of Mycobacterium tuberculosis strains and their efficiency for extrarespiratory infection. J Infect Dis 192, 2059-2065.

Hisert, K. B., Kirksey, M. A., Gomez, J. E., Sousa, A. O., Cox, J. S., Jacobs, W. R., Jr, Nathan, C. F. \& McKinney, J. D. (2004). Identification of Mycobacterium tuberculosis counterimmune (cim) mutants in immunodeficient mice by differential screening. Infect Immun 72, 5315-5321.

Jain, M., Petzold, C. J., Schelle, M. W., Leavell, M. D., Mougous, J. D., Bertozzi, C. R., Leary, J. A. \& Cox, J. S. (2007). Lipidomics reveals control of Mycobacterium tuberculosis virulence lipids via metabolic coupling. Proc Natl Acad Sci U S A 104, 5133-5138.

McAdam, R. A., Quan, S., Smith, D. A., Bardarov, S., Betts, J. C., Cook, F. C., Hooker, E. U., Lewis, A. P., Woollard, P. \& other authors (2002). Characterization of a Mycobacterium tuberculosis H37Rv transposon library reveals insertions in 351 ORFs and mutants with altered virulence. Microbiology 148, 2975-2986.

Nunn, P., Williams, B., Floyd, K., Dye, C., Elzinga, G. \& Raviglione, M. (2005). Tuberculosis control in the era of HIV. Nat Rev Immunol 5, 819-826.

Ormerod, L. P. (2005). Multidrug-resistant tuberculosis (MDR-TB): epidemiology, prevention and treatment. Br Med Bull 73-74, 17-24.

Parish, T. \& Stoker, N. G. (1998). Electroporation of mycobacteria. In Methods in Molecular Biology: Mycobacteria Protocols, pp. 129-144. Edited by T. Parish \& N. G. Stoker. Totowa, NJ: Humana Press.

Raetz, C. R. \& Roderick, S. L. (1995). A left-handed parallel $\beta$ helix in the structure of UDP-N-acetylglucosamine acyltransferase. Science 270, 997-1000.

Raviglione, M. C. (2003). The TB epidemic from 1992 to 2002. Tuberculosis (Edinb) 83, 4-14.

Rousseau, C., Neyrolles, O., Bordat, Y., Giroux, S., Sirakova, T. D., Prevost, M. C., Kolattukudy, P. E., Gicquel, B. \& Jackson, M. (2003). Deficiency in mycolipenate- and mycosanoate-derived acyltrehaloses enhances early interactions of Mycobacterium tuberculosis with host cells. Cell Microbiol 5, 405-415.

Sambrook, J., Fritsch, E. F. \& Maniatis, T. (1989). Molecular Cloning: a Laboratory Manual, 2nd edn. Cold Spring Harbor, NY: Cold Spring Harbor Laboratory.

Sassetti, C. M. \& Rubin, E. J. (2003). Genetic requirements for mycobacterial survival during infection. Proc Natl Acad Sci U S A 100, 12989-12994.

Sassetti, C. M., Boyd, D. H. \& Rubin, E. J. (2003). Genes required for mycobacterial growth defined by high density mutagenesis. Mol Microbiol 48, 77-84.

Sirakova, T. D., Thirumala, A. K., Dubey, V. S., Sprecher, H. \& Kolattukudy, P. E. (2001). The Mycobacterium tuberculosis pks2 gene encodes the synthase for the hepta- and octamethyl-branched fatty acids required for sulfolipid synthesis. J Biol Chem 276, 16833-16839.

Smith, R. J. \& Iden, S. S. (1981). Properties of calcium ionophoreinduced generation of superoxide anion by human neutrophils. Inflammation 5, 177-192.

Soto, C. Y., Cama, M., Gibert, I. \& Luquin, M. (2000). Application of an easy and reliable method for sulfolipid-1 detection in the study of its distribution in Mycobacterium tuberculosis strains. FEMS Microbiol Lett 187, 103-107.

Stewart, G. R., Patel, J., Robertson, B. D., Rae, A. \& Young, D. B. (2005). Mycobacterial mutants with defective control of phagosomal acidification. PLoS Pathog 1, 269-278.

Stokes, R. W. \& Doxsee, D. (1999). The receptor-mediated uptake, survival, replication, and drug sensitivity of Mycobacterium tuberculosis within the macrophage-like cell line THP-1: a comparison with human monocyte-derived macrophages. Cell Immunol 197, 1-9.

Stokes, R. W., Orme, I. M. \& Collins, F. M. (1986). Role of mononuclear phagocytes in expression of resistance and susceptibility to Mycobacterium avium infections in mice. Infect Immun 54, 811-819.

Stokes, R. W., Norris-Jones, R., Brooks, D. E., Beveridge, T. J., Doxsee, D. \& Thorson, L. M. (2004). The glycan-rich outer layer of the cell wall of Mycobacterium tuberculosis acts as an antiphagocytic capsule limiting the association of the bacterium with macrophages. Infect Immun 72, 5676-5686.

Stover, C. K., de la Cruz, V. F., Fuerst, T. R., Burlein, J. E., Benson, L. A., Bennett, L. T., Bansal, G. P., Young, J. F., Lee, M. H. \& other authors (1991). New use of BCG for recombinant vaccines. Nature 351, 456-460.

Takayama, K., Schnoes, H. K., Armstrong, E. L. \& Boyle, R. W. (1975). Site of inhibitory action of isoniazid in the synthesis of mycolic acids in Mycobacterium tuberculosis. J Lipid Res 16, 308-317.

Trivedi, O. A., Arora, P., Sridharan, V., Tickoo, R., Mohanty, D. \& Gokhale, R. S. (2004). Enzymic activation and transfer of fatty acids as acyl-adenylates in mycobacteria. Nature 428, 441-445.

Villeneuve, C., Etienne, G., Abadie, V., Montrozier, H., Bordier, C., Laval, F., Daffe, M., Maridonneau-Parini, I. \& Astarie-Dequeker, C. (2003). Surface-exposed glycopeptidolipids of Mycobacterium smegmatis specifically inhibit the phagocytosis of mycobacteria by human macrophages. Identification of a novel family of glycopeptidolipids. $J$ Biol Chem 278, 51291-51300.

Vuorio, R., Harkonen, T., Tolvanen, M. \& Vaara, M. (1994). The novel hexapeptide motif found in the acyltransferases LpxA and LpxD of lipid A biosynthesis is conserved in various bacteria. FEBS Lett 337, 289-292.

Waddell, S. J., Chung, G. A., Gibson, K. J. C., Everett, M. J., Minnikin, D. E., Besra, G. S. \& Butcher, P. D. (2005). Inactivation of polyketide synthase and related genes results in the loss of complex lipids in Mycobacterium tuberculosis H37Rv. Lett Appl Microbiol 40, 201-206.

Wenzel, C. Q., Daniels, C., Keates, R. A., Brewer, D. \& Lam, J. S. (2005). Evidence that $\mathrm{WbpD}$ is an $\mathrm{N}$-acetyltransferase belonging to the hexapeptide acyltransferase superfamily and an important protein for O-antigen biosynthesis in Pseudomonas aeruginosa PAO1. Mol Microbiol 57, 1288-1303.

Edited by: S. V. Gordon 